\title{
Hemodynamic effects of epinephrine in rats: evaluation by impedance cardiography
}

\author{
Turkseven CH, Pekoglu E, Buyukakilli B \\ Department of Biophysics, Faculty of Medicine, Mersin University, Mersin, Turkey. \\ bbuyukakilli@yahoo.com
}

\begin{abstract}
BACKGROUND AND OBJECTIVES: This study was aimed to examine how inotropic effects of intravenously injected epinephrine change thoracic impedance measurements and to reveal the possible effects of this change on other hemodynamic parameters by using the technique of impedance cardiography.

METHODS: 10 male Wistar Albino rats were divided into two equal groups: control and epinephrine. $0.2 \mathrm{mg} / \mathrm{kg}$ of epinephrine was administered to the rats in the epinephrine group via the tail vein. All hemodynamic parameters obtained by impedance cardiography [the base impedance $\left(Z_{0}\right)$, the maximum rate of change in impedance (dZmax/dt), the left ventricular ejection time (LVET), stroke volume (SV), cardiac output (CO), contractility index (IC), thoracic fluid content (TFC), heart rate (HR)] were recorded using the EBI 100C, DA 100 and ECG modules in the BIOPAC MP100 system.

RESULTS: CO ( $p \leq 0.05), \operatorname{HR}(p \leq 0.001), d Z_{\max } / d t(p \leq 0.05)$ and IC $(p \leq 0.05)$ increased statistically significantly in the epinephrine group compared to the control group. However, LVET $(p \leq 0.001)$ decreased statistically significantly in the epinephrine group compared to the control group.

CONCLUSION: Tachycardia was detected in the epinephrine group. There was an inverse correlation between LVET and dZmax/dt and IC. This is based on the fact that epinephrine increases inotropic effect (Tab. 2, Fig. 4, Ref. 30). Text in PDF www.elis.sk.

KEY WORDS: impedance cardiography, epinephrine, bioimpedance, hemodynamic.
\end{abstract}

\section{Introduction}

Cardiac arrest is a clinical situation characterized by a sudden cessation of the blood circulation due to lack of effective systolic contraction of the heart and sudden loss of consciousness due to inadequate cerebral blood flow (1). There are four cardiac arrest rhythms: ventricular fibrillation (VF), pulseless ventricular tachycardia (PVT), pulseless electrical activity (PEA) and cessation of the normal rhythmic contractions of the heart. According to the Advanced Cardiovascular Life Support (ACLS) guideline, drugs are used to stabilize the hemodynamics and to reproduce the rhythm providing perfusion and to improve cardiac output and organ perfusion (2). If the rhythmic contractions of the heart stop or pulseless electrical activity (PEA) occurs in patients with cardiac arrest, cardiopulmonary resuscitation (CPR) should be started immediately and epinephrine should be given (3). Epinephrine is a natural catecholamine which is alpha $(\alpha)$ - and beta $(\beta)$-adrenergic agonist. It is used primarily in cardiac arrest in emergency services. It increases coronary and cerebral blood flow through its $\alpha$-adrenergic effects during cardiac arrest. It also improves coronary artery perfusion

Department of Biophysics, Faculty of Medicine, Mersin University, Mersin, Turkey

Address for correspondence: B. Buyukakilli, Department of Biophysics, Faculty of Medicine, Mersin University TR-33343 Mersin, Turkey. Phone: +90.324 .3610001$ pressure by increasing peripheral vasoconstriction. $\beta$-adrenergic effects of epinephrine are increased heart rate, contractility and conduction velocity. It increases heart rate as a result of the increased rate of spontaneous depolarization in the sinoatrial node. Moreover, it increases conduction velocity in the AV node and shortens the refractory period in the cell membrane of ventricular muscle. However, it increases systemic vascular resistance, systolic and diastolic blood pressure, electrical activity in myocardial tissue, coronary and cerebral blood flow, myocardial contraction force and myocardial oxygen requirement (4). It has been seen that epinephrine was applied to improve hemodynamic parameters such as cardiac output (CO), mean arterial blood pressure (MAP) and systemic vascular resistance (SVR) and improved short-term clinical outcomes. Donnino's group in Boston analyzed the data from Get With the Guidelines-Resuscitation database (formerly National Registry of Cardiopulmonary Resuscitation, NRCPR). The database is sponsored by the American Heart Association (AHA) and contains prospective data from 570 American hospitals collected from 1 January 2000 to 19 November 2009. They found that in patients with non-shockable cardiac arrest in a hospital setting, earlier administration of epinephrine is associated with a higher probability of return to spontaneous circulation, survival in hospital, and neurologically intact survival (5). There are other studies supporting that epinephrine increases the survival of patients with cardiac arrest (6). Epinephrine is a widely used drug for the treatment of cardiac arrest. Because it facilitates the recovery of 
613-617

the patient by increasing diastolic and coronary perfusion pressure through its $\alpha$-agonist activity (7). Epinephrine binds to $\beta 1$ adrenergic receptors when released at the neuromuscular junction. Binding of epinephrine to its G-protein-linked receptor can lead to the activation of adenylyl cyclase. Adenylyl cyclase synthesizes cyclic adenosine monophosphate or cyclic AMP from adenosine triphosphate (ATP). Cyclic AMP activates protein kinases. Protein kinases activate $\mathrm{Ca}^{2+}$ release from intracellular $\mathrm{Ca}^{2+}$ stores by activating inositol-3 phosphate (IP3) receptors in the sarcoplasmic reticulum (8-15). A number of studies have been carried out on the effects of $\mathrm{Ca}^{2+}$, cyclic AMP and epinephrine on electrical and mechanical properties of the heart. It is concluded that the effects of epinephrine on activation of glycogenolysis and on the inotropic response of cardiac muscle are both mediated by the combined actions of $\mathrm{Ca}^{2+}$ and cyclic AMP. Since the inotropic response precedes the glycogenolytic response it is evident that increased energy metabolism is a consequence of increased heart work and not a causative factor. The epinephrine-induced increase in cytosolic $\mathrm{Ca}^{2+}$ levels increases the heart rate and cardiac contractility with actin-myosin interaction. It also alters the mechanical activity of the heart (16).

However, epinephrine also has side effects. Excessive calcium accumulation in cardiac muscle was first emphasized by Fleckenstein in 1971. Catecholamines open the L-type calcium channel more and thus permit more calcium entry into the cell. Fleckenstein defined experimental myocardial necrosis in response to high doses of epinephrine (17). Hypertension, tachycardia, myocyte damage and pulmonary edema may also occur during and after cardiopulmonary resuscitation (18). In addition, it has been shown that epinephrine increased mortality after brief asphyxial cardiac arrest. This is associated with left atrial hypertension as well as a decrease in left ventricular end-diastolic diameter (19). The accurate measurement of hemodynamic parameters of these side effects and their changes in response to physiological or pharmacological stimuli is an important factor in evaluation of the heart mechanical efficiency, especially for hemodynamic management of critically ill patients and evaluation of postoperative treatment progress in the intensive care units (20-21).

About 70 years ago, Kubicek et al. proposed impedance cardiography (ICG) as a noninvasive technique for continuous measurement of SV, cardiac output, and other hemodynamics for aerospace applications. In this method, pulsatile changes in the impedance of thorax are measured, which are mainly caused by the variations of aorta blood volume (22). In addition, reliable measurements of hemodynamic parameters during transient events (e.g., in atrial fibrillation or the presence of extrasystole) could allow quantitative estimation of cardiac performance when it is difficult or even impossible to apply well established, classical methods (20).

In the light of all these literature information, by using the technique of impedance cardiography which is a non-invasive method, we aimed to examine how inotropic effects of intravenously injected epinephrine change thoracic impedance measurements and to reveal the possible effects of this change on other hemodynamic parameters [stroke volume (SV), cardiac output (CO), contractility index (IC), total fluid content (TFC), heart rate (HR), left ventricular ejection time (LVET)].

\section{Material and methods}

\section{Animal preparation and experimental protocol}

10 male Wistar albino rats obtained from Mersin University Faculty of Medicine Experimental Animal Research and Training Unit were used in this study. Ethical approval has been received from the Medical Faculty Experimentation Ethics Committee of Mersin University. They were placed in plastic cages $(20 \times 35 \times 45 \mathrm{~cm}$ in size) with 5 rats in each cage. They were kept at seasonal conditions in a temperature-controlled room $\left(21 \pm 1^{\circ} \mathrm{C}\right)$ with a $12 \mathrm{~h}$ : $12 \mathrm{~h}$ light-dark cycle in accordance with the circadian rhythm during the experiment. Drinking water was poured from above, within glass bottles with a pipette tip, so that it could be reached when desired. Readymade food pellets were used and the cages were cleaned twiceweekly. The rats were allowed free access to their diet and water.

The experimental groups were planned in accordance with the following objectives:

1. The rats were anesthetized by intraperitoneal (i.p.) administration of ketamine hydrochloride $(90 \mathrm{mg} / \mathrm{kg})$ and xylazine hydrochloride $(7 \mathrm{mg} / \mathrm{kg})$. Then, they were placed in a supine position on the Electrically Heated Animal Operating Table (AOT0801-DC) in order to keep the rectal temperature at $37-38{ }^{\circ} \mathrm{C}$ during ECG and Impedance Cardiography recordings (Fig. 1).

Tab. 1. Study Groups.

\begin{tabular}{ll}
\hline $\mathrm{C}(\mathrm{n}=5)$ & Control \\
$\mathrm{E}(\mathrm{n}=5)$ & Epinephrine \\
\hline
\end{tabular}

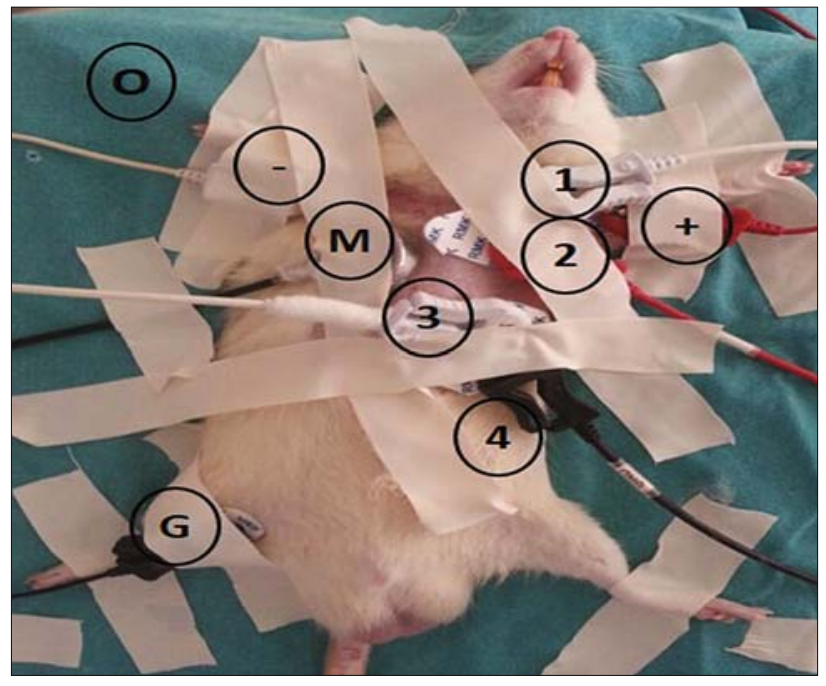

Fig. 1. The rats were placed in a supine position on the Electrically Heated Animal Operating Table $(O)$ in order to keep the rectal temperature at $37-38^{\circ} \mathrm{C}$. Impedance cardiography recordings were taken with the EBI100C module using superficial electrodes $(\mathrm{Ag} / \mathrm{AgCl})($ red numbers from 1 to 4$)$. Electric current $(70 \mathrm{kHz}, 2.5 \mathrm{~mA})$ was applied with the external electrodes ( 1 and 4 ) and the changes in voltage of the chest were measured by the internal electrodes ( 2 and 3 ). Superficial ECG electrodes were placed according to the lead I configuration in order to record ECG (right arm +, left arm - and right leg (ground) G) and the signals were recorded with the EKG100C module. The heart sounds were recorded with the DA100C module using the audio transducer microphone (M). 
2. $0.2 \mathrm{mg} / \mathrm{kg}$ of epinephrine was administered to the rats in the epinephrine group via the tail vein (23).

3. Simultaneous recordings were taken in the rats by superficial tetrapolar electrodes for impedance cardiography, by superficial ECG electrodes for electrocardiography and by superficial sound transducer for heart sounds. Then, bioimpedance and hemodynamic parameters were evaluated (Fig. 1).

In line with these objectives, 10 male Wistar Albino rats of $10-13$ weeks of age (220-320 g) included in the study were randomly divided into two groups as shown in Table 1 . Thoracic electrical bioimpedance records were analyzed in all of the rats in the control and epinephrine groups in the study.

\section{Impedance cardiography (ICG)}

In combination with heart sounds and ECG signals, ICG provides a continuous comprehensive hemodynamic profile for illness/treatment evaluations. All hemodynamic signals obtained by ICG, ECG and heart sounds, which are non-invasive methods, $\left(\mathrm{Z}_{0}\right.$ the base impedance, $\mathrm{dZmax} / \mathrm{dt}$ the maximum rate of change in impedance, the left ventricular ejection time (LVET), stroke volume (SV), cardiac output (CO), contractility index (IC), thoracic fluid content (TFC), heart rate (HR)) were simultaneously recorded in $60 \mathrm{sec}$ epochs using the EBI100C, DA100C with a physiological microphone and ECG modules in the BIOPAC MP100 system (Santa Barbara, USA) (Fig. 2) (24, 25). Impedance cardiography quantitatively determines hemodynamic parameters $(\mathrm{SV}, \mathrm{CO}$, IC, TFC) using base and maximum impedance changes $(26,27)$.

\section{Statistical analysis}

After the normal distribution of continuous measures was confirmed with the Shapiro-Wilk test, differences in parameters between groups were analyzed with the Independent-samples t-test. The Levene's test was used to check the homogeneity of the vari-

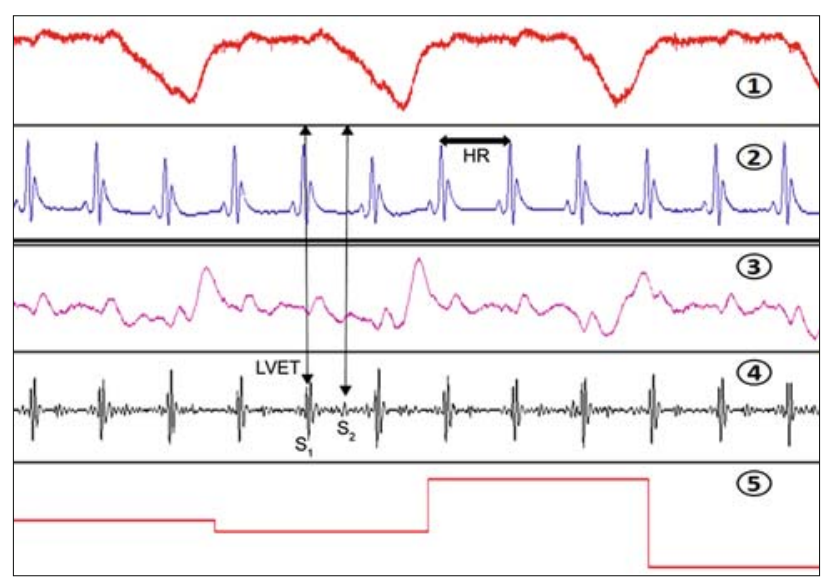

Fig. 2. Impedance cardiography, electrocardiogram and heart sounds were recorded simultaneously. Z0 base impedance (1). ECG (2). Heart rate was determined by measuring the time interval between $R-R$. The rate of change in impedance (3). Heart sounds (4). Left ventricular ejection time was determined as electromechanical systole time. As a result, left ventricular ejection time was measured as the time interval between the first heart sound (S1) and the second heart sound (S2). The maximum rate of change in impedance (5).
Tab. 2. Statistical results for impedance cardiography parameters (mean \pm standard deviation). The Independent-samples t-test was used to compare each parameter in the control and epinephrine groups.

\begin{tabular}{lccc}
\hline & Control (n=5) & Epinephrine $(\mathrm{n}=5)$ & $\mathrm{p}$ \\
\hline $\mathrm{Z}_{0}(\mathrm{ohm})$ & $81.454 \pm 5.549$ & $73.698 \pm 7.678$ & 0.437 \\
$\mathrm{dZ}_{\mathrm{Max}} / \mathrm{dt}(\mathrm{ohm} / \mathrm{s})$ & $5.768 \pm 0.851$ & $12.916 \pm 1.795$ & $0.007^{*}$ \\
$\mathrm{TFC}\left(\mathrm{kohm}^{-1}\right)$ & $12.489 \pm 0.784$ & $14.228 \pm 1.590$ & 0.336 \\
$\mathrm{IC}\left(\mathrm{s}^{-1}\right)$ & $0.069 \pm 0.005$ & $0.177 \pm 0.022$ & $0.007^{*}$ \\
LVET $(\mathrm{s})$ & $0.136 \pm 0.007$ & $0.095 \pm 0.003$ & $0.001^{*}$ \\
HR (beats/min) & $183.654 \pm 11.647$ & $305.576 \pm 16.090$ & $0.000^{*}$ \\
$\mathrm{SV}(\mathrm{ml} / \mathrm{beat})$ & $0.088 \pm 0.024$ & $0.139 \pm 0.016$ & 0.123 \\
$\mathrm{CO}(1 / \mathrm{min})$ & $0.015 \pm 0.004$ & $0.042 \pm 0.003$ & $0.002^{*}$ \\
\hline
\end{tabular}

Statistically significant difference was found in some parameters $(* p \leq 0.05)$. Z0-base impedance; dZMax/dt-maximum rate of change in impedance; TFC - thoracic fluid content; IC - contractility index; LVET - left ventricular ejection time; HR - heart rate; $\mathrm{SV}$ - stroke volume; $\mathrm{CO}$ - cardiac output

ances. Since the descriptive parameters were normally distributed, they were given as mean \pm standard deviation. SPSS 21 software package was used for the analysis. It is also utilized for graphics. $p$ values of less than 0.05 were considered as statistically significant.

\section{Results}

During the experiment, no rats died in the control and epinephrine groups. There was no statistically significant difference between the control and epinephrine groups in terms of $Z_{0}$, TFC and SV. On the other hand, SV and TFC increased non-significantly in the epinephrine group (Tab. 2). However, $\mathrm{dZ}_{\mathrm{Max}} / \mathrm{dt}, \mathrm{IC}$, $\mathrm{HR}$ and $\mathrm{CO}$ increased statistically significantly in the epinephrine group compared to the control group $(\mathrm{p} \leq 0.05)$ (Figs 3A-3B-3D and 4). Moreover, LVET decreased statistically significantly in the epinephrine group compared to the control group $(\mathrm{p} \leq 0.05)$ (Tab. 2) (Fig. 3C).

\section{Discussion}

Impedance cardiography is an easy and inexpensive method which gives information about hemodynamic parameters, needs no surgery, does not pose any risk to patients and has minimal technical requirements (27). Other methods that measure the same hemodynamic parameters are thermodilution, indicator dilution, and Fick method. These methods give accurate results of the measurements and require surgical operation. However, their use has recently been increasingly criticized due to uncertainty in their utility rates and costs (28). Recently, it has been shown that impedance cardiography can be used to determine liquid consistency and to predict cardiac decompensation (29). For this reason, the method we used in our study has an important place.

Chen et al (2010) evaluated the dose-response to epinephrine during cardiopulmonary resuscitation and identified what is the optimal dose of epinephrine in a rat cardiac arrest model. They showed that different doses of epinephrine produced the similar rate of restoration of spontaneous circulation, but that high-dose epinephrine inhibited the recovery of spontaneous ventilation and caused relative bradycardia after CPR in an asphyxial rat model. They reported that low and medium doses of epinephrine were 
$613-617$
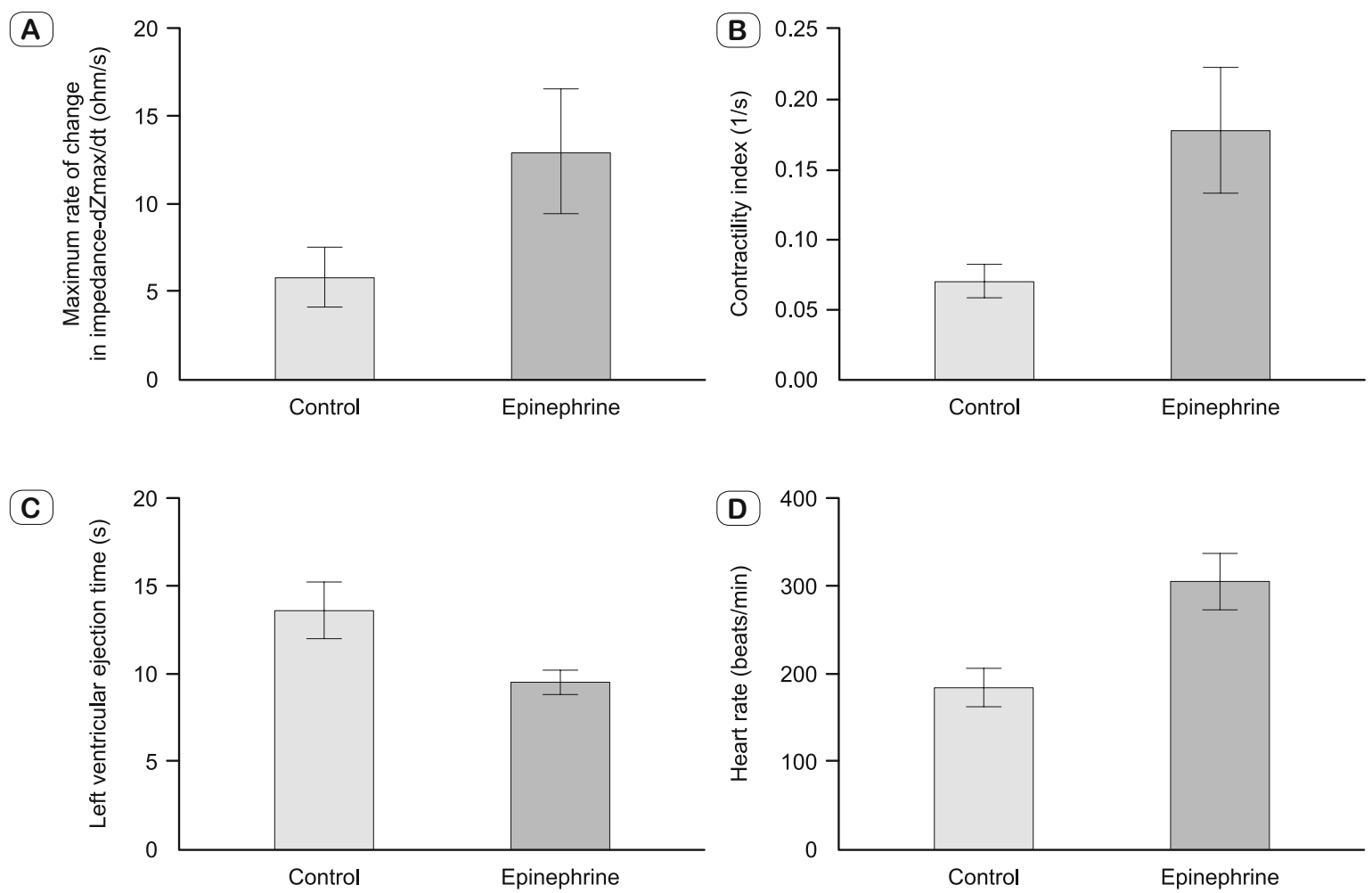

Fig. 3. When impedance cardiography parameters were compared between groups (mean \pm standard deviation), (A) maximum rate of change in impedance, (B) contractility index and (D) heart rate increased statistically significantly in the epinephrine group compared to the control group (p $\leq \mathbf{0 . 0 5})$. (C) Left ventricular ejection time decreased statistically significantly in the epinephrine group compared to the control group $(\mathbf{p} \leq \mathbf{0 . 0 5})$.

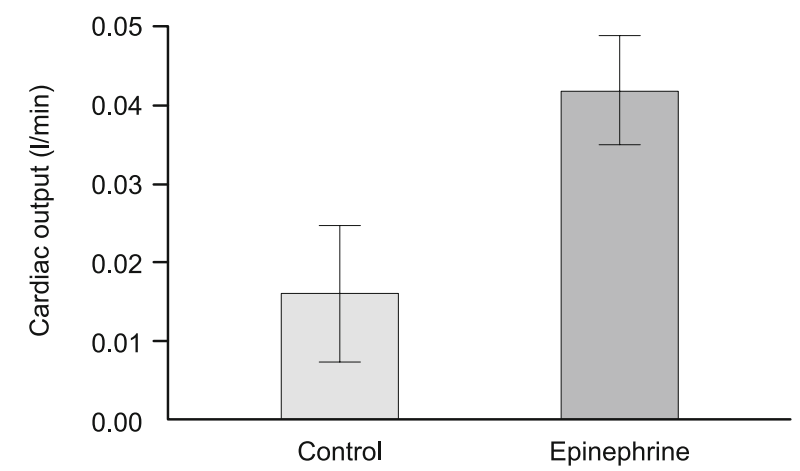

Fig. 4. Cardiac output (mean \pm standard deviation) increased statistically significantly in the epinephrine group compared to the control group (p $\leq$ 0.05).

more optimal for CPR in a rat asphyxial cardiac arrest model. Therefore, in our study we used the medium dose to show hemodynamic effects of epinephrine in rats by ICG.

It was shown in our study that the effect of epinephrine on cardiac function was compatible with other studies $(4,16,30)$. In a study conducted, impedance reduction and left ventricular filling pressure were found to be inversely correlated. This is based on the fact that fluid accumulation increases the electrical conductivity across the lungs (29). In this study, although it was not statistically significant, the decrease in the base impedance $\left(\mathrm{Z}_{0}\right)$ value in the epinephrine group supports this result. In another study, epinephrine has been found to increase contractility. In fact, this demonstrates that epinephrine is not only a potent stimulant of cardiac chronotropic activity, but also a strong stimulus of myocardial contractility (4). In our study, in the epinephrine group it was found that the contractility index increased but left ventricular ejection time decreased. This also suggests that epinephrine increases inotropic effect.

In another study, epinephrine was found to increase stroke volume, heart rate, and cardiac output (30). This result is inconsistent with the result that although epinephrine increased stroke volume in our study, it did not make a statistically significant difference between the groups. However, it increased statistical3ly significantly heart rate and maximum rate of change in impedance and as a result cardiac output is measured by multiplying heart rate by stroke volume. Therefore, this supports that epinephrine increased cardiac output.

\section{Conclusion}

Indications for the use of epinephrine are based on a limited number of animal studies. In addition, there is a small number of studies on impedance cardiography, which is a non-invasive meth- 
od, in which we will be able to monitor the effect of epinephrine in emergency situations. In our study, hemodynamic effects of medium dose epinephrine administration in experimental animals were shown with impedance cardiography. As a result, epinephrine induced inotropic effects and thus increased the maximum rate of change in impedance depending on the increase in heart rate and cardiac output. However, an inverse correlation between the contractility index and the left ventricular ejection time is based on the fact that epinephrine increases coronary perfusion. In the light of these data, prospective studies are needed to determine the appropriate dose, timing and indications for epinephrine.

\section{References}

1. Kazak $\mathbf{Z}$ and Ökten F. Pathophysiology of Cardiac Arrest. Turkiye Klinikleri J Surg Med Sci 2007; 3 (6): 10-14.

2. Cummins RO. Pharmacology II: Agents to Control Rate and Rhythm. In Advanced Cardiovascular Life Support (ACLS). American Heart Association $2003 ; 239-259$.

3. ECC Committee, Subcommittees and Task Forces of the American Heart Association. American Heart Association Guidelines for Cardiopulmonary Resuscitation and Emergency Cardiovascular Care. Circulation 2005; 112: 1- 211.

4. Unverir P, Karcıoğlu O, Ayrık C. Usage of The Advanced Cardiovascular Life Support Drugs In Sudden Cardiac Arrest. DEÜ Tıp Fakültesi Dergisi 2006; 2: 125-133.

5. Donnino MW, Salciccioli JD, Howell MD et al. Time to administration of epinephrine and outcome after in-hospital cardiac arrest with nonshockable rhythms: retrospective analysis of large in-hospital data registry. BMJ 2014; 348: 1-9.

6. Liu H, Sangkum L, Liu G, Green M, Li M, Kaye A. Effects of epinephrine on angiogenesis-related gene expressions in cultured rat cardiomyocytes. JBR 2016; 30 (5): 380-385.

7. Ali B, Zafari AM. Narrative review: cardiopulmonary resuscitation and emergency cardiovascular care: review of the current guidelines. Ann Intern Med 2007; 147: 171-179.

8. Treinys R, Jurevicius J. L-type $\mathrm{Ca}^{2+}$ channels in the heart: structure and regulation. Medicina (Kaunas) 2008; 44: 491-499.

9. Dibb KM, Graham HK, Venetucei LA, Eisner DA, Trafford AW. Analysis of cellular calcium fuxes in cardiac muscle to understand calcium homeostasis in the heart. Cell Calcium 2007; 42: 503-512.

10. Pitt GS, Dun W, Boyden PA. Remodeled cardiac calcium channels. J Mol Cell Cardiol 2006; 41: 373-388.

11. Bodi I, Mikala G, Koch SE, Akhter SA, Schwartz A. The L-type calcium channel in the heart: the beat goes on. J Clin Invest 2005; 115 : 3306-3317.

12. Petrovic MM, Vales K, Putnikovic B, Djulejic V, Mitrovic DM. Ryanodine receptors, voltage-gated calcium channels and their relationship with protein kinase A in the myocardium. Physiol Res 2008; 57: 141-149.

13. Orchard C, Brette F. t-Tubules and sarcoplasmic reticulum function in cardiac ventricular myocytes. Cardiovasc Res 2008; 77: 237-244.
14. Hund TJ, Ziman AP, Lederer WJ, Mohler PJ. The cardiac IP3 receptor: uncovering the role of "the other" calcium-release channel. J Mol Cell Cardiol 2008; 45: 159-161.

15. Diaz ME, Graham HK, O'Neill SC, Trafford AW, Eisner DA. The control of sarcoplasmic reticulum Ca content in cardiac muscle. Cell Calcium 2005; 38: 391-396.

16. Williamson JR, Schaffer S. Epinephrine, cyclic AMP, calcium, and myocardial contractility. Recent Adv Stud Cardiac Struct Metab 1976; 9: 205-223.

17. Fleckenstein A. Specific inhibitors and promoters of calcium action in the excitation-contraction coupling of heart muscle and their role in the prevention of production of myocardial lesions. In:Harris P, Opie LH (Eds). Calcium and the heart. Academic press, London, 1971; 135-142.

18. Shah S, Gopalakrishnan S, Apuya J, Shah S, Martin T. Use of intralipid in an infant with impending cardiovascular collapse due to local anesthetic toxicity. J Anesth 2009; 23: 439-441.

19. McCaul CL, McNamara PJ, Engelberts D et al. Epinephrine increases mortality after brief asphyxial cardiac arrest in an in vivo rat model. Anesth Analg 2006; 102: 542-548.

20. Cybulski G, Strasz A, Niewiadomski W, Gasiorowska A. Impedance cardiography: Recent advancements. Cardiol J 2012; 19: 550-556.

21. Konings MK, Goovaerts HG, Roosendaal MR et al. A new electric method for non-invasive continuous monitoring of stroke volume and ventricular volume-time curves. Biomed Eng Online 2012; 11: 51.

22. Yazdanian H, Mahnam A, Edrisi M, Esfahani MA. Design and Implementation of a Portable Impedance Cardiography System for Noninvasive Stroke Volume Monitoring. J Med Signals Sens 2016; 6 (1): 47-56.

23. Chen MH, Lu JY, Xie L, Zheng JH, Song FQ. What is the optimal dose of epinephrine during cardiopulmonary resuscitation in a rat model? The American Journal of Emergency Med 2010; 28 (3) : 284-290.

24. De Pasquale MJ and Fossa AA. Use of bioimpedance for measuring cardiac output in the conscious dog. Drug Dev Res 1996; 38: 105-113.

25. Bernstein DP. A new stroke volume equation for thoracic electrical bioimpedance: theory and rational. Crit Care Med 1986; 14: 904-909.

26. van de Water JM, Mount BE, Chandra KM, Mitchell BP, Woodruff TA, Dalton ML. TFC (thoracic fluid content): A new parameter for assessment of changes in chest fluid volume. Am Surg 2005; 71: 81-86.

27. Buyukakilli B, Gurul S, Citırık D, Hallioglu O, Ozeren M, Tasdelen B. Determination of the effects of pulmonary arterial hypertension and therapy on the cardiovascular system of rats by impedance cardiography. Croat Med J 2014; 55: 498-506.

28. Scherhag A, Kaden JJ, Kentschke E, Sueselbeck T, Borggrefe M. Comparison of impedance cardiography and thermodilution-derived measurements of stroke volume and cardiac output at rest and during exercise testing. Cardiovasc Drugs Ther 2005; 19: 141-147.

29. Becher J, Kaufmann SG, Paule S et al. Device-based impedance measurement is a useful and accurate tool for direct assessment of intrathoracic fluid accumulation in heart failure. Europace 2010; 12 (5): 731-740.

30. Liu CA, Sui J, Coté CJ, Anderson TA. The Use of Epinephrine in Caudal Anesthesia Increases Stroke Volume and Cardiac Output in Children. Reg Anesth Pain Med 2016; 41 (6): 780-786. 